\title{
Mobility and Einstein Relation for a tagged particle in asymmetric mean zero random walk with simple exclusion
}

\author{
Michail Loulakis \\ Statistical Laboratory, Centre for Mathematical Sciences, Wilberforce Road, Cambridge CB3 OWB, United Kingdom \\ Received 5 May 2003; received in revised form 29 June 2004; accepted 6 July 2004 \\ Available online 22 January 2005
}

\begin{abstract}
The subject of this article is to study the asymptotic velocity of a tracer particle in a mean zero asymmetric simple exclusion system in $\mathbb{Z}^{d}$ (a diffusive, non-reversible particle system), slightly perturbed from equilibrium. The perturbation can be thought of to be induced by a small uniform external field $E$. The leading linear order term in $E$ of the velocity is called the mobility of the particle and the Einstein relation states that it coincides with the self-diffusion matrix of the tracer particle in equilibrium. We compute the mobility when $d \geqslant 3$, and we show that such a relation fails to hold for all mean-zero asymmetric simple exclusion systems. The method we use to compute the mobility is quite general and applicable to a wide range of models.

(c) 2005 Elsevier SAS. All rights reserved.
\end{abstract}

\section{Résumé}

On s'intéresse à la vitesse asymptotique d'une particule marquée dans un processus d'exclusion simple centré asymétrique, soumise à un champ de force externe $E$. La mobilité de la particule marquée, estimation au premier ordre de la vitesse pour de petites valeurs de $E$, coincide avec la matrice d'auto-diffusion, relation dite d'Einstein. Dans cet article, on calcule la mobilité pour $d \geqslant 3$ et l'on montre que la relation d'Einstein cesse d' être valide pour tous les processus d'exclusion simple centrés asymétriques. La méthode utilisée pour calculer la mobilité est très génerale et peut s'appliquer à une grande classe de modèles. (C) 2005 Elsevier SAS. All rights reserved.

\section{Introduction}

Consider a diffusive particle system in equilibrium, tag a particle and let $D$ be its self-diffusion coefficient. Suppose now the system is perturbed by a small field that affects the dynamics of the tagged particle but not that of its environment. More precisely, the type of perturbation we consider is the following: if $\mathbb{P}^{0}$ is the process in equilibrium with infinitesimal generator $L$ and $X_{t}$ denotes the position of the tagged particle at time $t$, then the

E-mail address: michail@statslab.cam.ac.uk (M. Loulakis). 
perturbed process $\mathbb{P}^{V}$ is a measure absolutely continuous to $\mathbb{P}^{0}$ when we only consider paths up to a finite time $t$, with a Radon-Nikodym derivative of the form

$$
\exp \left(V\left(X_{t}\right)-V\left(X_{0}\right)-\int_{0}^{t} \mathrm{e}^{-V\left(X_{s}\right)} L \mathrm{e}^{V\left(X_{s}\right)} \mathrm{d} s\right) .
$$

In physical terms, $V\left(X_{t}\right)$ represents the work done on the system by the external field up to time $t$.

If under the new dynamics the tagged particle has a preferred direction of motion, for instance in the case of a uniform external field $\left(V\left(X_{t}\right)=\alpha \cdot X_{t}\right)$, it is of interest to examine whether it moves with a ballistic velocity $v(\alpha)$. In that case linear response theory predicts that the mobility of the tagged particle defined as $M_{i j}=\left.\frac{\partial v_{i}(\alpha)}{\partial \alpha_{j}}\right|_{\alpha=0}$ coincides with the self-diffusion matrix $D$. Such a relation is usually referred to as the Einstein relation and it is believed to be generally valid, at least for reversible particle systems.

A natural class of models for which one would like to establish the validity of such a relation is simple exclusion processes on $\mathbb{Z}^{d}$. The motion (in the scaling limit) of a tracer particle when these models are in equilibrium is by now well understood. Saada [15] proved a law of large numbers for the position of a tracer particle and Kipnis and Varadhan [4], Varadhan [18] and Sethuraman, Varadhan and Yau [16] have proved an invariance principle for its fluctuations in the symmetric, mean-zero asymmetric, and general asymmetric in $d \geqslant 3$ case, respectively.

In computing the mobility of the tagged particle we are faced with the difficulty that the particle system in the presence of the field evolves out of equilibrium, and there is not much explicit information available for the invariant states of the system under the new dynamics. Consequently, the physically proper order of limits, first the scaling limit to compute the drift, then $\alpha \rightarrow 0$ to compute the mobility presents a hard problem.

Ferrari, Goldstein and Lebowitz [1] proposed rescaling the external field simultaneously with space and time as an alternative. This 'weak asymmetry' approach was successfully carried out by Lebowitz and Rost in [11]. Landim, Olla and Volchan [9] studied the motion of an asymmetric tracer particle for the 1-dimensional nearest neighbor symmetric simple exclusion process. This is the only case where invariant measures for the process 'as seen from the particle' are explicitly known [1]. Relating the motion of the particle to the diffusion of a particle system with zero range interaction they obtained results for a wide class of non-equilibrium initial configurations, including the validity of the Einstein relation. In both these articles, the symmetry under time reversal is substantially used.

The validity of the Einstein relation was proved in [13] for all symmetric simple exclusion processes in three or more dimensions. However, the reversibility of the system is very mildly used there, and the method of proof allows for applications to different particle systems including non-reversible ones.

In this article we apply this method to compute the mobility of a tagged particle for the mean-zero asymmetric simple exclusion process in $d \geqslant 3$, a diffusive non-reversible particle system. It turns out, even though it requires some work to prove so, that at least at sufficiently low particle densities the Einstein relation is always violated, which hints to the fundamental role of reversibility in the validity of such a relation. As corollaries to our results we also show that the self-diffusion coefficient of a tagged particle in mean-zero asymmetric simple exclusion is always strictly greater than the one in its symmetric counterpart, and that as a function of the particle density $\rho$ it is of class $C^{\infty}$ at zero as well, slightly extending the main result in [7] to the mean-zero asymmetric case. Finally, we discuss briefly the applicability of the method to other particle systems and give examples where it can be successfully used.

\section{Notation and results}

Let us fix a finite range probability measure $p(\cdot)$ on $\mathbb{Z}^{d}$, with $p(0)=0$. Consider now an initial configuration of particles on $\mathbb{Z}^{d}$, such that each site is occupied by at most one particle. The simple exclusion process describes the 
evolution where particles perform random walks on $\mathbb{Z}^{d}$ with transition probability $p(x, y)=p(y-x)$ and interact through hard-core exclusion, i.e. jumps on sites that are already occupied are suppressed.

A natural state space for the process is $\mathbb{X}=\{0,1\}^{\mathbb{Z}^{d}}$. If $\xi \in \mathbb{X}$ and $x \in \mathbb{Z}^{d}$, then $\xi(x)$ is 1 or 0 according to whether the site $x$ is occupied or not. The generator $L$ of this Markov process on $\mathbb{X}$ acts on local functions (i.e. functions depending on $\xi$ through only a finite number of coordinates) as:

$$
L f(\xi)=\sum_{x, y} p(y-x) \xi(x)(1-\xi(y))\left(f\left(\xi^{x, y}\right)-f(\xi)\right),
$$

where:

$$
\xi^{x, y}(z)= \begin{cases}\xi(z) & \text { if } z \neq x, y \\ \xi(x) & \text { if } z=y \\ \xi(y) & \text { if } z=x\end{cases}
$$

In order to avoid degeneracies we will assume that the random walk in $\mathbb{Z}^{d}$ with one step transition probabilities $p(y-x)$ is irreducible, i.e. $\{x: p(x)>0\}$ generates the group $\mathbb{Z}^{d}$. We will also assume that $p(\cdot)$ has zero average, i.e. $\sum x p(x)=0$. The symmetric and the anti-symmetric part of $p(\cdot)$ will be denoted by $a(\cdot)$ and $b(\cdot)$ respectively:

$$
a(x)=\frac{p(x)+p(-x)}{2}, \quad b(x)=\frac{p(x)-p(-x)}{2} .
$$

As the number of particles is conserved by the dynamics, there is a family of invariant measures: $P_{\rho}(0 \leqslant \rho \leqslant 1)$, defined as Bernoulli products of parameter $\rho$ over the sites of $\mathbb{Z}^{d}$ are invariant and ergodic for the evolution of the processes (cf. [12], Chapter VIII for a proof).

In order to study the motion of the tagged particle, consider an initial configuration $\xi$, chosen according to $P_{\rho}$ conditioned to have a particle at the origin. Tag this particle and denote by $X_{t}$ its position at time $t$. Even though $X_{t}$ is not Markov due to the presence of the environment, $\left(X_{t}, \xi_{t}\right)$ clearly is. It is useful to consider the process of 'the environment as seen from the particle' $\eta_{t}: \eta_{t}(x)=\xi_{t}\left(X_{t}+x\right)$. This is itself a Markov process, whose infinitesimal generator is given by $\mathcal{L}=\mathcal{L}^{\text {ex }}+\mathcal{L}^{\text {sh }}$, where:

$$
\begin{aligned}
& \mathcal{L}^{\mathrm{ex}} f(\eta)=\sum_{x, y \neq 0} p(y-x) \eta(x)(1-\eta(y))\left(f\left(\eta^{x, y}\right)-f(\eta)\right), \\
& \mathcal{L}^{\mathrm{sh}} f(\eta)=\sum_{z} p(z)(1-\eta(z))\left(f\left(\tau_{z} \eta\right)-f(\eta)\right) .
\end{aligned}
$$

(Here $\tau_{z} \eta$ stands for the configuration obtained from $\eta$ by moving the tagged particle to $z$, then shifting the entire configuration by $-z$.) $\mathcal{L}^{\text {ex }}$ corresponds to jumps of the environment, and $\mathcal{L}^{\text {sh }}$ takes into account the jumps of the tagged particle. A simple computation shows that $\mu_{\rho}:=P_{\rho}(\cdot \mid \xi(0)=1)$ is invariant for the process $\eta_{t}$, and Saada [15] proved that $\mu_{\rho}$ is ergodic.

Local functions form a core of the generator $\mathcal{L}$ (cf, [12], I.2-3), and it is also simple to check that the adjoint of $\mathcal{L}$ in $L^{2}\left(\mu_{\rho}\right)$ is the generator $\mathcal{L}^{*}$ of the 'environment as seen from the particle' process associated to the law $p^{*}(x)=p(-x)$. Hence, if the transition law of the underlying walk is $a(\cdot)$, then the corresponding generator $\mathcal{S}\left(=\frac{1}{2}\left(\mathcal{L}+\mathcal{L}^{*}\right)\right.$ on local functions) extends to a self-adjoint operator in $L^{2}\left(\mu_{\rho}\right)$.

For local functions $f$ we can define the Dirichlet form $\mathcal{D}_{\rho}(f)=\langle f,(-\mathcal{L}) f\rangle_{\rho}$. It is easy to verify that $\mathcal{D}_{\rho}(f)=$ $\langle f,(-\mathcal{S}) f\rangle_{\rho}=\mathcal{D}_{\rho}^{\mathrm{ex}}(f)+\mathcal{D}_{\rho}^{\mathrm{sh}}(f)$, where

$$
\begin{aligned}
& \mathcal{D}_{\rho}^{\mathrm{ex}}(f)=\frac{1}{4} \int \sum_{x, y \neq 0} a(y-x)\left(f\left(\eta^{x y}\right)-f(\eta)\right)^{2} \mathrm{~d} \mu_{\rho}, \quad \text { and } \\
& \mathcal{D}_{\rho}^{\mathrm{sh}}(f)=\frac{1}{2} \int \sum_{z} a(z)(1-\eta(z))\left(f\left(\tau_{z} \eta\right)-f(\eta)\right)^{2} \mathrm{~d} \mu_{\rho} .
\end{aligned}
$$


We can also define the seminorm $\|\cdot\|_{1, \rho}$ by: $\|f\|_{1, \rho}^{2}=\mathcal{D}_{\rho}(f)$, and the equivalence relation $f \sim g \Leftrightarrow\|f-g\|_{1, \rho}$ $=0$. It is immediate from the definition that $\|\cdot\|_{1, \rho}$ satisfies the parallelogram identity. Therefore, by completing the quotient space with respect to $\|\cdot\|_{1, \rho}$ we obtain a Hilbert space, which will be denoted by $H_{1, \rho}$. The dual of $H_{1, \rho}$ with respect to the standard inner product in $L^{2}\left(\mu_{\rho}\right)$, is another Hilbert space which will be denoted by $H_{-1, \rho}$. In particular if $\psi \in L^{2}\left(\mu_{\rho}\right) \cap H_{-1, \rho}$,

$$
\|\psi\|_{-1, \rho}^{2}=\sup _{f \text { local }}\left\{2\langle f, \psi\rangle_{\rho}-\|f\|_{1, \rho}^{2}\right\} .
$$

A similar Sobolev space $H_{1,0, \rho}$ can be defined by completing with respect to the seminorm $\|\cdot\|_{1,0, \rho}$, defined by: $\|f\|_{1,0, \rho}^{2}=\mathcal{D}_{\rho}^{\mathrm{ex}}(f)$. The dual of this space will be denoted by $H_{-1,0, \rho}$.

Let $N_{t}^{z}$ be the process that counts the jumps of the tagged particle by $z . N_{t}^{z}$ is a Poisson process with variable rate $\lambda(z, t)=p(z)\left(1-\eta_{t}(z)\right)$. If $J_{t}^{z}$ is the compensated Poisson process associated to $N_{t}^{z}$ then it follows immediately that for any vector $\ell$ in $\mathbb{R}^{d}$ :

$$
X_{t} \cdot \ell=\sum(\ell \cdot z) N_{t}^{z}=\sum_{z}(\ell \cdot z) J_{t}^{z}+\int_{0}^{t} \omega_{\ell}\left(\eta_{s}\right) \mathrm{d} s,
$$

where

$$
\omega_{\ell}(\eta)=\sum_{z} p(z)(z \cdot \ell)(1-\eta(z)) .
$$

Varadhan [18] showed that the rightmost term in (2) can be approximated by a martingale and he established an invariance principle for the position of a tagged particle, i.e. the convergence in the Skorokhod space of $\varepsilon X_{t \varepsilon^{-2}}$, as $\varepsilon \rightarrow 0$ to a diffusion.

The variance $D(\rho)$ of the limiting diffusion can be explicitly described in terms of the inner product in $H_{-1, \rho}$ : Let us denote the reflection operator on $\mathbb{X}$ by

$$
R \eta(z)=\eta(-z) .
$$

The action of $R$ is naturally extended to functions and measures on $\mathbb{X}$. Clearly, $\mu_{\rho}$ is $R$-invariant and $R^{2}=\mathrm{Id}$. Thus, $R$ preserves the norm and is self-adjoint in $L^{2}\left(\mu_{\rho}\right)$. Furthermore, the following commutation relation can be readily verified:

$$
R \mathcal{L}=\mathcal{L}^{*} R
$$

In particular, $R$ commutes with $\mathcal{S}$. Hence, $R$ also preserves the norms $\|\cdot\|_{1, \rho}$ and $\|\cdot\|_{-1, \rho}$. Similarly, $R$ preserves $\|\cdot\|_{1,0, \rho}$, and $\|\cdot\|_{-1,0, \rho}$, because it commutes with the part of $\mathcal{S}$ that takes into account only the jumps of the environment. The variance $D(\rho)$ can now be described as the symmetric $d \times d$ matrix such that $\forall \ell \in \mathbb{R}^{d}$ (cf. [8]):

$$
\ell \cdot D(\rho) \ell=(1-\rho) \sum_{z} p(z)(z \cdot \ell)^{2}+2 \lim _{\lambda \downarrow 0}\left\langle(\lambda-\mathcal{L})^{-1} \omega_{\ell}, R \omega_{\ell}\right\rangle .
$$

We next want to study the motion of the tagged particle under the dynamics that is generated by $\mathcal{L}_{\alpha}=\mathcal{L}^{\mathrm{ex}}+\mathcal{L}_{\alpha}^{\mathrm{sh}}$, where:

$$
\mathcal{L}_{\alpha}^{\mathrm{sh}}=\sum_{z} p(z) \mathrm{e}^{\alpha \cdot z}(1-\eta(z))\left(f\left(\tau_{z} \eta\right)-f(\eta)\right)
$$

In the notation of the introduction this perturbation of the original dynamics corresponds to work $V\left(X_{t}\right)=\alpha \cdot X_{t}$ (e.g. a homogeneous field $\alpha$ acting on a particle of unit charge). We will denote by $\mathbb{P}^{\alpha, \eta}$ the measure on the space of càdlàg paths on $\mathbb{X}$ that corresponds to this process started from the configuration $\eta$, and we define $\mathbb{P}^{\alpha, \rho}=$ $\int \mathbb{P}^{\alpha, \eta} \mathrm{d} \mu_{\rho}(\eta) . \mathbb{E}^{\alpha, \eta}$ and $\mathbb{E}^{\alpha, \rho}$ will be respectively used to denote expectations under these measures. 
The rate of $N_{t}^{z}$ under $\mathbb{P}^{\alpha, \eta}$ is $\lambda_{\alpha}(z, t)=p(z) \mathrm{e}^{\alpha \cdot z}\left(1-\eta_{t}(z)\right)$. Therefore:

$$
X_{t} \cdot \ell=\sum(z \cdot \ell) N_{t}^{z}=\sum_{z}(\ell \cdot z) M_{t}^{z}+\int_{0}^{t} \omega_{\ell}^{\alpha}\left(\eta_{s}\right) \mathrm{d} s
$$

where now

$$
\omega_{\ell}^{\alpha}(\eta)=\sum_{z} p(z) \mathrm{e}^{\alpha \cdot z}(z \cdot \ell)(1-\eta(z))
$$

and the $\mathbb{P}^{\alpha, \eta}$-martingales $M_{t}^{z}$ are the compensated Poisson processes associated to $N_{t}^{z}$. Note that jumps in the direction of $\alpha$ are now favoured, so we expect the tagged particle to pick up a velocity rather than diffuse. This suggests a hyperbolic scaling $(z \mapsto \varepsilon z, t \mapsto \varepsilon t)$ in order to yield nontrivial results. After rescaling, the martingale term in (6) converges to zero $\mathbb{P}^{\alpha, \eta}$-a.s., so essentially we need to study the time averages of $\omega_{\ell}^{\alpha}\left(\eta_{t}\right)$. The difficulty arises by the fact that the initial state $\mu_{\rho}$ is not invariant under the new dynamics so the system evolves out of equilibrium. However, as in [13] we can prove that the limiting behavior of these time averages is determined up to the first order of the magnitude of $\alpha$, so the mobility of the tagged particle can be defined. But before we can give the precise statement of the results in this paper we need to introduce some notation.

Let $\mathcal{C}$ be the space of real-valued continuous functions on $\mathbb{X}$, and $\mathcal{M}$ be the space of probability measures on $\mathbb{X}$ equipped with the topology of weak convergence of measures. For $f \in \mathcal{C}$ and $\mu \in \mathcal{M}$ we will denote $\int f(\eta) \mathrm{d} \mu(\eta)$ either by $\langle f, \mu\rangle$ or by $E^{\mu}[f] . \lambda_{\rho}^{*}(f)$ will stand for the principal eigenvalue of the self-adjoint operator $\mathcal{S}+f$ in $L^{2}\left(\mu_{\rho}\right)$. Let also $h_{\alpha} \in \mathcal{C}$ be:

$$
h_{\alpha}(\eta)=\sum_{z} p(z)\left((\alpha \cdot z) \mathrm{e}^{\alpha \cdot z}-\mathrm{e}^{\alpha \cdot z}+1\right)(1-\eta(z)) .
$$

Note that $h_{\alpha}$ is nonnegative and has a quadratic behavior for small values of $\alpha$. Let now $\mathcal{I}_{\alpha}=\left\{\mu \in \mathcal{M}:\left(\mathcal{L}_{\alpha}\right)^{*} \mu=\right.$ $0\}$ be the set of invariant states of the process, and

$$
\mathcal{A}_{\alpha, \rho}=\left\{\mu \in \mathcal{I}_{\alpha}:\langle f, \mu\rangle \leqslant\left\langle h_{\alpha}, \mu\right\rangle+\lambda_{\rho}^{*}(f), \forall f \in \mathcal{C}\right\} .
$$

By Theorem 2 in [13] the sets $\mathcal{A}_{\alpha, \rho}$ are non-empty, and their elements assign probability 1 to configurations with average particle density $\rho$ when $d \geqslant 3$.

The first theorem provides estimates for the displacement of the tagged particle:

Theorem 1. In any dimension $d$, for every $\ell \in \mathbb{R}^{d}, t \geqslant 0$ we have $\mathbb{P}^{\alpha, \rho}$-a.s.

$$
t \inf _{\mu \in \mathcal{A}_{\alpha, \rho}} E^{\mu}\left[\omega_{\ell}^{\alpha}\right] \leqslant \liminf _{\varepsilon \rightarrow 0} \varepsilon\left(X_{t \varepsilon^{-1}} \cdot \ell\right) \leqslant \limsup _{\varepsilon \rightarrow 0} \varepsilon\left(X_{t \varepsilon^{-1}} \cdot \ell\right) \leqslant t \sup _{\mu \in \mathcal{A}_{\alpha, \rho}} E^{\mu}\left[\omega_{\ell}^{\alpha}\right] .
$$

The next result concerns the asymptotic behavior of $E^{\mu}\left[\omega_{\ell}^{\alpha}\right]$ for $\mu \in \mathcal{A}_{\alpha, \rho}$, as $\alpha \rightarrow 0$.

Theorem 2. If $d \geqslant 3$, then for any $\ell \in \mathbb{R}^{d}$ we have:

$$
\frac{1}{|\alpha|} \sup _{\mu \in \mathcal{A}_{\alpha, \rho}}\left|E^{\mu}\left[\omega_{\ell}^{\alpha}\right]-(1-\rho) \sum_{z} p(z)(\ell \cdot z)(\alpha \cdot z)+\lim _{\lambda \downarrow 0}\left\langle(\lambda-\mathcal{L})^{-1} \omega_{\ell},(1-R) \omega_{\alpha}\right\rangle_{\rho}\right| \longrightarrow 0,
$$

as $\alpha \rightarrow 0$.

In view of Theorems 1 and 2 the first order term in $\alpha$ of the drift in the direction $\ell$ is:

$$
v(\alpha) \cdot \ell=(1-\rho) \sum_{z} p(z)(\ell \cdot z)(\alpha \cdot z)-\lim _{\lambda \downarrow 0}\left\langle(\lambda-\mathcal{L})^{-1} \omega_{\ell},(1-R) \omega_{\alpha}\right\rangle_{\rho},
$$


so the mobility matrix $M$ is given by:

$$
M_{i j}(\rho)=(1-\rho) \sum_{z} p(z) z_{i} z_{j}-\lim _{\lambda \downarrow 0}\left\langle(\lambda-\mathcal{L})^{-1} \omega_{e_{i}},(1-R) \omega_{e_{j}}\right\rangle_{\rho} .
$$

Let us recall formula (5) for the self-diffusivity now, and define:

$$
\Delta_{\ell}(\rho)=\ell(D(\rho)-M(\rho)) \ell=\lim _{\lambda \downarrow 0}\left((\lambda-\mathcal{L})^{-1} \omega_{\ell},(1+R) \omega_{\ell}\right)_{\rho} .
$$

Let us also define the odd and even parts of $\omega_{\ell}$ under $R$ :

$$
\begin{aligned}
& \psi_{\ell}(\eta)=\frac{1-R}{2} \omega_{\ell}=\sum_{x} a(x)(x \cdot \ell)(1-\eta(x)), \\
& \phi_{\ell}(\eta)=\frac{1+R}{2} \omega_{\ell}(\eta)=\sum_{x} b(x)(x \cdot \ell)(1-\eta(x)) .
\end{aligned}
$$

It is easy to see that if $\ell \in \mathbb{R}^{d}$ is such that the support of $b(\cdot)$ is not included in the hyperplane $x \cdot \ell=0$, then $\phi_{\ell} \neq 0$.

It is not clear at this point that $\Delta_{\ell}(\rho)$ is not identically zero. After all, $\omega_{\ell}(\eta)$ is the image of the function $F(x, \eta)=x$ under the generator of the process $\left(X_{t}, \eta_{t}\right)$, and so is $\psi_{\ell}(\eta)$ in the symmetric case. It could be possible that as $\lambda$ approaches zero, $(\lambda-\mathcal{L})^{-1} \omega_{\ell}-(\lambda-\mathcal{S})^{-1} \psi_{\ell}$ weakly converges to zero in $H_{1, \rho}$, in which case $\Delta_{\ell}(\rho)$ would be zero since $(\lambda-\mathcal{S})^{-1} \psi_{\ell}$ is antisymmetric under $R$. Theorem 3 shows that this is not the case.

\section{Theorem 3.}

$$
\Delta_{\ell}(\rho)=4 \rho(1-\rho)\left\|\phi_{\ell}\right\|_{-1,0, \frac{1}{2}}^{2}+\mathrm{o}(\rho), \quad \text { as } \rho \rightarrow 0 .
$$

Hence, for all mean zero asymmetric simple exclusion processes the self diffusivity is different from the mobility at sufficiently small densities $\rho$, and the Einstein relation does not hold.

The rest of this article is organized as follows: In Section 3 we prove Theorems 1 and 2. Some estimates on the generator which are needed there, as well as for the proof of Theorem 3 are proved in Section 4 . Section 5 contains the proof of Theorem 3. In Section 6 we make some remarks on properties of the self-diffusion matrix $D(\rho)$ in the mean zero asymmetric case that can be concluded from the results of this paper. We also discuss the generalization of this method for computing the mobility in other systems.

\section{Computation of the mobility matrix}

As we mentioned in the introduction, reversibility is very mildly used in [13] for the computation of the mobility. It is not surprising thus that the proofs of Theorems 1 and 2 almost go through verbatim in the non-reversible case as well. We will only give their outline here.

Proof of Theorem 1. The processes $\mathbb{P}^{\alpha, \eta}$ and $\mathbb{P}^{0, \eta}$ can be written as a Girsanov type transform of each other. If $\mathcal{F}_{t}:=\sigma\left(\eta_{s} ; s \leqslant t\right)$ and we define:

$$
\Psi_{t}(\alpha) \stackrel{\text { def }}{=} \exp \left(\sum_{z}(\alpha \cdot z) N_{t}^{z}-\int_{0}^{t} \sum_{z} \lambda(z, s)\left(\mathrm{e}^{\alpha \cdot z}-1\right) \mathrm{d} s\right),
$$

then $\Psi_{t}(\alpha)$ is a $\mathbb{P}^{0, \eta}$-martingale adapted to $\mathcal{F}_{t}$, and $\mathbb{P}^{\alpha, \eta}$ is given by:

$$
\left.\frac{\mathrm{d} \mathbb{P}^{\alpha, \eta}}{\mathrm{d} \mathbb{P}^{0, \eta}}\right|_{\mathcal{F}_{t}}=\Psi_{t}(\alpha)
$$


Using elementary inequalities and Lemma 7.2 in Appendix 1 of [3] one can prove (cf. [13]) the following large deviations upper bounds:

1. For every $\varepsilon>0$ there exists a strictly positive constant $C(\varepsilon)$ such that:

$$
\mathbb{P}^{\alpha, \eta}\left(\sum_{z}(\ell \cdot z) M_{t}^{z}>t \varepsilon\right) \leqslant \mathrm{e}^{-C(\varepsilon) t}
$$

2. Let $f$ be a local function on $\mathbb{X}$. Then, for every $\varepsilon>0$ there exist strictly positive constants $C(\varepsilon), A$ such that:

$$
\mathbb{P}^{\alpha, \eta}\left(\int_{0}^{t} \mathcal{L}_{\alpha} f\left(\eta_{s}\right) \mathrm{d} s>t \varepsilon\right) \leqslant A \mathrm{e}^{-C(\varepsilon) t} .
$$

3. Let $f \in \mathcal{C}$. For every $\varepsilon>0$ there exists a strictly positive constant $C(\varepsilon)$ such that:

$$
\mathbb{P}^{\alpha, \rho}\left(\frac{1}{t} \int_{0}^{t} f\left(\eta_{s}\right)-h_{\alpha}\left(\eta_{s}\right) \mathrm{d} s>\lambda_{\rho}^{*}(f)+\varepsilon\right) \leqslant \mathrm{e}^{-C(\varepsilon) t} .
$$

Recall Eq. (2) for the position of the tagged particle. After rescaling we have:

$$
\varepsilon\left(X_{t \varepsilon^{-1}} \cdot \ell\right)=\varepsilon \sum_{z}(\ell \cdot z) M_{t \varepsilon^{-1}}^{z}+\varepsilon \int_{0}^{t \varepsilon^{-1}} \omega_{\ell}^{\alpha}\left(\eta_{s}\right) \mathrm{d} s .
$$

Clearly, the martingale term on the right-hand side goes to zero by (10). On the other hand, if we define the random measures

$$
v_{t}:=\frac{1}{t} \int_{0}^{t} \delta_{\eta_{s}} \mathrm{~d} s \in \mathcal{M} \quad\left(\text { so for } f \in \mathcal{C}: \frac{1}{t} \int_{0}^{t} f\left(\eta_{s}\right) \mathrm{d} s=\left\langle f, v_{t}\right\rangle\right),
$$

it follows from (11), (12) and the compactness of $\mathbb{X}$ that with $\mathbb{P}^{\alpha, \rho}$-probability 1 , any weak limit of $v_{t}$ is in $\mathcal{A}_{\alpha, \rho}$. Thus, for any continuous function $f$ (and in particular for $\omega_{\ell}^{\alpha}$ ) with $\mathbb{P}^{\alpha, \rho}$-probability 1 we have:

$$
t \inf _{\mu \in \mathcal{A}_{\alpha, \rho}} E^{\mu}[f] \leqslant \liminf _{\varepsilon \rightarrow 0} \varepsilon \int_{0}^{t \varepsilon^{-1}} f\left(\eta_{s}\right) \mathrm{d} s \leqslant \limsup _{\varepsilon \rightarrow 0} \varepsilon \int_{0}^{t \varepsilon^{-1}} f\left(\eta_{s}\right) \mathrm{d} s \leqslant t \sup _{\mu \in \mathcal{A}_{\alpha, \rho}} E^{\mu}[f] .
$$

Proof of Theorem 2. We can rewrite $\omega_{\ell}^{\alpha}$ as:

$$
\omega_{\ell}^{\alpha}=\omega_{\ell}+(1-\rho) \sum_{z} p(z)(\alpha \cdot z)(\ell \cdot z)+R_{1}-R_{2}(\eta),
$$

where

$$
\begin{aligned}
& R_{1}=(1-\rho) \sum_{z} p(z)(\ell \cdot z)\left(\mathrm{e}^{\alpha \cdot z}-(\alpha \cdot z)-1\right), \\
& R_{2}(\eta)=\sum_{z} p(z)\left(\mathrm{e}^{\alpha \cdot z}-1\right)(\ell \cdot z)(\eta(z)-\rho) .
\end{aligned}
$$

Clearly, $\left\|R_{1}\right\| \leqslant C|\alpha|^{2}$, and by formula (37) in [13] we have:

$$
\sup _{\nu \in \mathcal{A}_{\alpha, \rho}}\left|\left\langle R_{2}, \nu\right\rangle\right| \leqslant C|\alpha|^{2} .
$$


Hence, we need to show that:

$$
\frac{1}{|\alpha|} \sup _{\nu \in \mathcal{A}_{\alpha, \rho}}\left|E^{v}\left[\omega_{\ell}\right]+\lim _{\lambda \downarrow 0}\left\langle(\lambda-\mathcal{L})^{-1} \omega_{\ell},(1-R) \omega_{\alpha}\right\rangle_{\rho}\right| \longrightarrow 0 .
$$

Instead of studying the asymptotics of $E^{v}\left[\omega_{\ell}\right]$ for $v \in \mathcal{A}_{\alpha, \rho}$ as $\alpha \rightarrow 0$ directly, it is easier to look at $E^{v}[\mathcal{L} g]$, where $g$ is a local function, and approximate $\omega_{\ell}$ by a function in the range of the generator.

$$
\begin{aligned}
\langle\mathcal{L} g, v\rangle & =\left\langle\left(\mathcal{L}-\mathcal{L}_{\alpha}\right) g, v\right\rangle \quad\left(v \in \mathcal{I}_{\alpha}\right) \\
& =\left\langle\left(\mathcal{L}-\mathcal{L}_{\alpha}\right) g, \mu_{\rho}\right\rangle+\left\langle\left(\mathcal{L}-\mathcal{L}_{\alpha}\right) g-E^{\mu \rho}\left[\left(\mathcal{L}-\mathcal{L}_{\alpha}\right) g\right], v\right\rangle .
\end{aligned}
$$

The expectation under $\mu_{\rho}$ of $\left(\mathcal{L}-\mathcal{L}_{\alpha}\right) g$ can easily be computed with a change of variables $\eta \mapsto \tau_{z} \eta$, that changes the measure $(1-\eta(z)) \mathrm{d} \mu_{\rho}$ to $(1-\eta(-z)) \mathrm{d} \mu_{\rho}$ :

$$
\begin{aligned}
E^{\mu_{\rho}}\left[\left(\mathcal{L}-\mathcal{L}_{\alpha}\right) g\right] & =\sum_{z} p(z)\left(1-\mathrm{e}^{\alpha \cdot z}\right) \int g(\eta)[(1-\eta(-z))-(1-\eta(z))] \mathrm{d} \mu_{\rho} \\
& =\left\langle g,(1-R) \sum_{z} p(z)\left(\mathrm{e}^{\alpha \cdot z}-1\right)(1-\eta(z))\right\rangle_{\rho} .
\end{aligned}
$$

As far as the last term in relation (14) is concerned, notice that it is of the form: $R_{\alpha}(\eta)=\sum_{z} p(z) c_{z}(\alpha) w_{z}(\eta)$, where $c_{z}(\alpha)=1-\mathrm{e}^{\alpha \cdot z}(=\mathrm{O}(|\alpha|))$ and $w_{z}$ are $\mu_{\rho}$-mean zero local functions. We have:

$$
\sup _{\nu \in \mathcal{A}_{\alpha, \rho}}\left\langle R_{\alpha}, \nu\right\rangle \leqslant\left\|h_{\alpha}\right\|_{\infty}+\lambda_{\rho}^{*}\left(\sum_{z} p(z) c_{z}(\alpha) w_{z}(\eta)\right) \leqslant\left\|h_{\alpha}\right\|_{\infty}+\sum_{z} p(z) \lambda_{\rho}^{*}\left(c_{z}(\alpha) w_{z}\right) .
$$

By Lemma 2 in [13], if $d \geqslant 3$ and $f \in \mathcal{C}(\mathbb{X})$ then

$$
\lim _{\gamma \rightarrow 0} \gamma^{-1} \lambda_{\rho}^{*}(\gamma f)=\left\langle f, \mu_{\rho}\right\rangle
$$

Hence, by (14)-(17), if $d \geqslant 3$ and $g$ is a local function, we have:

$$
\lim _{\alpha \rightarrow 0} \frac{1}{|\alpha|} \sup _{\nu \in \mathcal{A}_{\alpha, \rho}}\left|\langle\mathcal{L} g, v\rangle-\left\langle g,(1-R) \omega_{\alpha}\right\rangle_{\mu_{\rho}}\right|=0
$$

The rest of the proof consists of approximating $\omega_{\ell}$ by a function in the range of the generator and controlling the error. This is done respectively, in the Lemmata 1 and 2 that follow.

Let $\Lambda$ be a finite subset in $\mathbb{Z}_{*}^{d}$, denote by $\mu_{\rho}^{\Lambda}$ the restriction of $\mu_{\rho}$ to configurations in $\Lambda$, and consider the canonical measures:

$$
v_{\Lambda, K}:=\mu_{\rho}^{\Lambda}\left(\cdot \mid \sum_{x \in \Lambda} \eta(x)=K\right)
$$

Let also $\mathcal{C}_{0}$ denote the space of local functions $\psi$ whose expectation under any canonical measure $v_{\Lambda, K}$, such that $\Lambda$ contains the support of $\psi$, is zero. Examples of such functions are the $\omega_{\ell}$, as well as functions of the form $\mathcal{L} g$, where $g$ is a local function.

Lemma 1. If $\psi$ is a local function in $\mathcal{C}_{0}$, then for every $\varepsilon>0$ there exist local functions, $g_{\varepsilon}, r_{\varepsilon}$ such that

$$
\psi=\mathcal{L} g_{\varepsilon}+r_{\varepsilon}, \quad \text { and } \quad\left\|r_{\varepsilon}\right\|_{-1,0, \rho}<\varepsilon
$$

Lemma 2. If $\psi$ is a local function in $\mathcal{C}_{0}$, then:

$$
\limsup _{\alpha \rightarrow 0} \frac{1}{|\alpha|} \sup _{\nu \in \mathcal{A}_{\alpha, \rho}}|\langle\psi, v\rangle| \leqslant C\|\psi\|_{-1,0, \rho}
$$


Lemma 1 is proved in [6] (cf. Theorem 4.2 there) for the symmetric simple exclusion. The proof in the mean zero asymmetric case, which follows closely the original one, is postponed until the next chapter, where the essential ingredients are stated and verified in our case. Lemma 2 relies upon (17), and is the content of (35) in [13].

\section{Estimates on the generator}

For $n \geqslant 0$ we denote by $\mathcal{E}_{n}$ the subsets of $\mathbb{Z}_{*}^{d}$ with cardinality $n$, and define $\mathcal{E}=\bigcup_{n} \mathcal{E}_{n}$. We also set $\chi(\rho)=$ $\rho(1-\rho)$, and define for every $A \in \mathcal{E}$ :

$$
\xi_{A}(\eta)=\prod_{x \in A} \frac{\eta(x)-\rho}{\sqrt{\chi(\rho)}} .
$$

By convention $\xi_{\emptyset}=0$. It is not hard to see that the family of local functions: $\left\{\xi_{A} ; A \in \mathcal{E}\right\}$ form an orthonormal basis for $L^{2}\left(\mu_{\rho}\right)$ and provide the orthogonal decomposition:

$$
L^{2}\left(\mu_{\rho}\right)=\bigoplus_{n \geqslant 0} \overline{G_{n}}, \quad \text { where } G_{n}=\operatorname{span}\left\{\xi_{A} ; A \in \mathcal{E}_{n}\right\}
$$

If we denote by $\pi_{n}$ the orthogonal projection on $G_{n}$ then for a local function $f$ on $\mathbb{X}$ we have:

$$
f=\sum_{n} \pi_{n} f=\sum_{n} \sum_{A \in \mathcal{E}_{n}} \mathfrak{f}(A) \xi_{A} .
$$

The Fourier coefficients $\mathfrak{f}(\cdot)$ in this expansion depend of course on $\rho$.

Let us consider now an abstract (real) Hilbert space $\mathfrak{H}$, with a complete orthonormal basis $\left\{e_{A}\right\}$, indexed by $\mathcal{E}$. We will denote by $\mathfrak{G}_{n}$ the linear span of the basis elements indexed by $\mathcal{E}_{n}$.

The mapping $f \mapsto \mathfrak{f}=\sum \mathfrak{f}(A) e_{A}$ maps elements of $G_{n}$ to elements in $\mathfrak{G}_{n}$ and clearly:

$$
\langle f, g\rangle_{\rho}=\langle\mathfrak{f}, \mathfrak{g}\rangle_{\mathfrak{H}}=\sum_{A \in \mathcal{E}} \mathfrak{f}(A) \mathfrak{g}(A) .
$$

In other words $f \mapsto \mathfrak{f}$ induces a unitary isomorphism between $L^{2}\left(\mu_{\rho}\right)$ and $\mathfrak{H}$. The generator $\mathcal{L}$ as well as the associated Dirichlet form can also be represented through this isomorphism. This approach offers two important advantages: first, the dependence on the density $\rho$ of inner products in $L^{2}\left(\mu_{\rho}\right)$ is incorporated in the Fourier coefficients, and second, the Fourier coefficients $\mathfrak{f}(\cdot)$ of a function $f \in G_{n}$, being functions from $\mathcal{E}_{n}$ to $\mathbb{R}$, are not significantly different from symmetric functions of $n \mathbb{Z}^{d}$-valued variables. Hence standard Fourier analysis techniques can be applied. This approach, originally presented in [10], has also led to quite fruitful applications in $[16,6-8]$.

If $A$ is a subset of $\mathbb{Z}_{*}^{d}$ and $x, y \in \mathbb{Z}_{*}^{d}$, we denote by $A^{x, y}$ the set:

$$
A^{x, y}= \begin{cases}A \backslash\{x\} \cup\{y\} & \text { if } x \in A, y \notin A, \\ A \backslash\{y\} \cup\{x\} & \text { if } y \in A, x \notin A, \\ A & \text { otherwise. }\end{cases}
$$

We also denote by $\tau_{z} A$ the set:

$$
\tau_{z} A= \begin{cases}A-z & \text { if } z \notin A, \\ (A-z)^{0,-z} & \text { if } z \in A .\end{cases}
$$

Therefore, in order to obtain $\tau_{z} A$ when $z \in A$ we first translate $A$ by $-z$ (getting a set that contains the origin), then remove the origin and add $\{-z\}$.

For a local function $f$ it is a matter of a simple computation to express the Fourier coefficients of $\mathcal{L} f$. If we define: 


$$
\begin{aligned}
\mathfrak{L}_{0} \mathfrak{f}(A) & =\sum_{x \in A, y \notin A} a(y-x)\left(\mathfrak{f}\left(A^{x, y}\right)-\mathfrak{f}(A)\right), \\
\mathfrak{L}_{0}^{b} \mathfrak{f}(A)= & \sum_{\substack{y \notin A, y \neq 0 \\
x \in A}} b(y-x)\left(\mathfrak{f}\left(A^{x, y}\right)-\mathfrak{f}(A)\right), \\
\mathfrak{L}_{0}^{-} \mathfrak{f}(A)= & \sum_{\substack{x, y \notin A \\
x, y \neq 0}} b(y-x)(\mathfrak{f}(A \cup\{y\})-\mathfrak{f}(A \cup\{x\})), \\
\mathfrak{L}_{0}^{+} \mathfrak{f}(A)= & \sum_{x, y \in A} b(y-x)(\mathfrak{f}(A \backslash\{y\})-\mathfrak{f}(A \backslash\{x\})),
\end{aligned}
$$

then $\mathcal{L}^{\text {ex }}$ is represented on $\mathfrak{H}$ by:

$$
\mathfrak{L}^{\mathrm{ex}}(\rho)=\mathfrak{L}_{0}+(1-2 \rho) \mathfrak{L}_{0}^{b}+\sqrt{\chi(\rho)}\left(\mathfrak{L}_{0}^{-}+\mathfrak{L}_{0}^{+}\right) .
$$

Similarly, $\mathcal{L}^{\text {sh }}$ is represented on $\mathfrak{H}$ by:

$$
\mathfrak{L}^{\mathrm{sh}}(\rho)=(1-\rho) \mathfrak{L}_{\tau}^{1}+\rho \mathfrak{L}_{\tau}^{2}+\sqrt{\chi(\rho)}\left(\mathfrak{L}_{\tau}^{-}+\mathfrak{L}_{\tau}^{+}\right),
$$

where:

$$
\begin{aligned}
& \mathfrak{L}_{\tau}^{1} \mathfrak{f}(A)=\sum_{z \notin A} p(z)\left(\mathfrak{f}\left(\tau_{z} A\right)-\mathfrak{f}(A)\right), \\
& \mathfrak{L}_{\tau}^{2} \mathfrak{f}(A)=\sum_{z \in A} p(z)\left(\mathfrak{f}\left(\tau_{z} A\right)-\mathfrak{f}(A)\right), \\
& \mathfrak{L}_{\tau}^{+} \mathfrak{f}(A)=\sum_{z \in A} p(z)\left(\mathfrak{f}(A \backslash\{z\})-\mathfrak{f}\left(\tau_{z}(A \backslash\{z\})\right)\right), \\
& \mathfrak{L}_{\tau}^{-} \mathfrak{f}(A)=\sum_{z \notin A} p(z)\left(\mathfrak{f}(A \cup\{z\})-\mathfrak{f}\left(\tau_{z}(A \cup\{z\})\right)\right) .
\end{aligned}
$$

Functions in $\mathfrak{G}_{n}$ are said to be of degree $n$. It is worth noticing that $\mathfrak{L}_{0}, \mathfrak{L}_{0}^{b}, \mathfrak{L}_{\tau}^{1}, \mathfrak{L}_{\tau}^{2}$ all preserve the degree of a function, while $\mathfrak{L}_{0}^{+}, \mathfrak{L}_{\tau}^{+}$increase the degree by 1 , and $\mathfrak{L}_{0}^{-}, \mathfrak{L}_{\tau}^{-}$decrease the degree by 1 .

A Dirichlet form associated to $\mathfrak{L}$ can be defined for the linear span of the basis elements of $\mathfrak{H}$. The resulting semi-norm will be denoted by $\|\mathfrak{f}\|_{1, \rho}$, and clearly if $\mathfrak{g}(\cdot)$ are the Fourier coefficients of a local function $g$, then $\|\mathfrak{g}\|_{1, \rho}=\|g\|_{1, \rho}$. The Sobolev spaces $\mathfrak{H}_{1, \rho}$ and $\mathfrak{H}_{-1, \rho}$ can be defined in the same fashion as $H_{1, \rho}$ and $H_{-1, \rho}$.

Similar Sobolev spaces can be induced by the symmetric positive operator $\mathfrak{L}_{0}$ and the corresponding norms will be denoted by $\|\cdot\|_{1, \text { env }}$ and $\|\cdot\|_{-1, \text { env }}$. It is worth observing that unlike $\|\mathfrak{f}\|_{ \pm 1, \rho}$, the norms $\|\mathfrak{f}\|_{ \pm 1, \text { env }}$ do not depend on $\rho$ (provided of course $\mathfrak{f}(\cdot)$ do not), and as $\mathfrak{L}_{0}$ is degree preserving, we have:

$$
\|\mathfrak{f}\|_{1, e n v}^{2}=\sum_{n \geqslant 0}\left\|\pi_{n} \mathfrak{f}\right\|_{1, e n v}^{2}, \quad \text { and } \quad\|\mathfrak{f}\|_{-1, e n v}^{2}=\sum_{n \geqslant 0}\left\|\pi_{n} \mathfrak{f}\right\|_{-1, e n v}^{2} .
$$

Hereafter, $C$ will be used to denote a constant that only depends on the law $p(\cdot)$ of the random walk. In particular it is independent of the density $\rho$ or the degree of a function.

Theorem 4. If $d \geqslant 3$ we have:

(i) Let $\mathfrak{L}^{+}=\mathfrak{L}_{0}^{+}+\mathfrak{L}_{\tau}^{+}$(resp. $\mathfrak{L}^{-}=\mathfrak{L}_{0}^{-}+\mathfrak{L}_{\tau}^{-}$) be the piece of the generator that increases (resp. decreases) the degree of a function by 1 . Then for $\mathfrak{f} \in \mathfrak{G}_{n}, \mathfrak{g} \in \mathfrak{G}_{n+1}$ (resp. $\mathfrak{g} \in \mathfrak{G}_{n-1}$ ) we have:

$$
\left|\left\langle\mathfrak{g}, \mathfrak{L}^{ \pm} \mathfrak{f}\right\rangle_{\mathfrak{H}}\right| \leqslant C \sqrt{n}\|\mathfrak{f}\|_{1, \text { env }}\|\mathfrak{g}\|_{1, \text { env }} .
$$


(ii) If $\mathfrak{f}, \mathfrak{g} \in \mathfrak{G}_{n}$, then:

$$
\begin{aligned}
& \left|\left\langle\mathfrak{g}, \mathfrak{L}_{0}^{b} \mathfrak{f}\right\rangle_{\mathfrak{H}}\right| \leqslant C n\|\mathfrak{f}\|_{1, \text { env }}\|\mathfrak{g}\|_{1, \text { env }}, \\
& \left|\left\langle\mathfrak{g}, \mathfrak{L}_{\tau}^{1} \mathfrak{f}\right\rangle_{\mathfrak{H}}\right| \leqslant C n\|\mathfrak{f}\|_{1, \text { env }}\|\mathfrak{g}\|_{1, \text { env }}, \\
& \left|\left\langle\mathfrak{g}, \mathfrak{L}_{\tau}^{2} \mathfrak{f}\right\rangle_{\mathfrak{H}}\right| \leqslant C \sqrt{n}\|\mathfrak{f}\|_{1, \text { env }}\|\mathfrak{g}\|_{1, \text { env }} .
\end{aligned}
$$

Note 1. If $\|\cdot\|_{1, \text { env }}$ is replaced by $\|\cdot\|_{1}$ in the right-hand side of (19)-(22) the estimates hold in any dimension with bounds that do not depend on $n$, as one expects from the sector condition for the generator that holds with a bound uniform in $\rho$. A proof of this fact can be found in [17].

Proof. The first estimate follows immediately by Lemma (4.1) and formula (3.14) in [16] and Lemma (5.1) of [6].

Recall that since $\mathfrak{g}, \mathfrak{f} \in \mathfrak{G}_{n}$ :

$$
\left\langle\mathfrak{g}, \mathfrak{L}_{0}^{b} \mathfrak{f}\right\rangle_{\mathfrak{H}}=\sum_{|A|=n} \mathfrak{g}(A) \sum_{\substack{y \notin A, y \neq 0 \\ x \in A}} b(y-x)\left(\mathfrak{f}\left(A^{x, y}\right)-\mathfrak{f}(A)\right) .
$$

The functions $\mathfrak{g}(\cdot)$ and $\mathfrak{f}(\cdot)$ are defined on $\mathcal{E}_{n}$, but can be viewed as symmetric functions of $n \mathbb{Z}^{d}$-valued variables. As such they are only defined on

$$
\mathcal{X}_{n}=\left\{\left(x_{1}, \ldots, x_{n}\right) \in\left(\mathbb{Z}^{d}\right)^{n}: x_{i} \neq 0, x_{i} \neq x_{j}, \forall i, j \in\{1, \ldots, n\} \text { with } i \neq j\right\},
$$

but they can be extended to $\left(\mathbb{Z}^{d}\right)^{n}$ by defining them to be zero outside $\mathcal{X}_{n}$. Using the convention:

$$
f\left(x_{1}, \ldots, x_{n}\right)= \begin{cases}\mathfrak{f}\left(\left\{x_{1}, \ldots, x_{n}\right\}\right) & \text { if }\left(x_{1}, \ldots, x_{n}\right) \in \mathcal{X}_{n}, \\ 0 & \text { otherwise }\end{cases}
$$

the right-hand side of (23) becomes:

$$
\begin{aligned}
& \frac{1}{n !} \sum_{x_{1}, \ldots, x_{n}} g\left(x_{1}, \ldots, x_{n}\right) \sum_{j=1}^{n} \sum_{y \neq 0, x_{1}, \ldots, x_{n}} b\left(y-x_{j}\right)\left(f\left(x_{1}, \ldots, y, \ldots, x_{n}\right)-f\left(x_{1}, \ldots, x_{j}, \ldots, x_{n}\right)\right) \\
& =\frac{1}{n !} \sum_{x_{1}, \ldots, x_{n}} g\left(x_{1}, \ldots, x_{n}\right) \sum_{j=1}^{n} \sum_{y} b\left(y-x_{j}\right) f\left(x_{1}, \ldots, y, \ldots, x_{n}\right) \\
& \quad+\frac{1}{n !} \sum_{x_{1}, \ldots, x_{n}} g\left(x_{1}, \ldots, x_{n}\right) f\left(x_{1}, \ldots, x_{n}\right) \sum_{j=1}^{n} \sum_{y} b\left(y-x_{j}\right) \\
& =\frac{1}{n !} \sum_{x_{1}, \ldots, x_{n}} g\left(x_{1}, \ldots, x_{n}\right) \sum_{j=1}^{n} \sum_{y} b\left(y-x_{j}\right) f\left(x_{1}, \ldots, y, \ldots, x_{n}\right)-\sum_{|A|=n} \mathfrak{g}(A) \mathfrak{f}(A)\left(\sum_{x \in A} b(x)\right) .
\end{aligned}
$$

In these steps we made use of the antisymmetry of $b$, as well as its consequence $\sum_{x} b(x)=0$ and of the fact that $f$ vanishes outside $\mathcal{X}_{n}$.

The last term in (25) is easy to estimate. If we define $W_{1}(A)=\sum_{x \in A} a(x)$, then by Lemma (3.7) in [16] we can find a constant $C$, such that for every $\mathfrak{u} \in \mathfrak{G}_{n}$ :

$$
\sum_{|A|=n} W_{1}(A) \mathfrak{u}^{2}(A) \leqslant C\|\mathfrak{u}\|_{1, e n v}^{2} .
$$

Using the fact that $|b(x)| \leqslant a(x)$, the Cauchy-Schwarz inequality and the preceding estimate:

$$
\left|\sum_{|A|=n} \mathfrak{g}(A) \mathfrak{f}(A)\left(\sum_{x \in A} b(x)\right)\right| \leqslant C\|\mathfrak{g}\|_{1, \text { env }}\|\mathfrak{f}\|_{1, \text { env }} .
$$


If we define the Fourier transform of a function $\psi$ on $\left(\mathbb{Z}^{d}\right)^{n}$ by:

$$
\hat{\psi}\left(\theta_{1}, \ldots, \theta_{n}\right)=\frac{1}{\sqrt{n !}} \sum_{\left(x_{1}, \ldots, x_{n}\right) \in\left(\mathbb{Z}^{d}\right)^{n}} \psi\left(x_{1}, \ldots, x_{n}\right) \mathrm{e}^{\mathrm{i}\left(\theta_{1} \cdot x_{1}+\cdots+\theta_{n} \cdot x_{n}\right)},
$$

then the first term in $(25)$ can be written as:

$$
\frac{1}{(2 \pi)^{n d}} \int_{\left(\mathbb{T}^{d}\right)^{n}} \overline{\hat{g}}\left(\theta_{1}, \ldots, \theta_{n}\right) \hat{f}\left(\theta_{1}, \ldots, \theta_{n}\right)\left(\sum_{j=1}^{n} \hat{b}\left(\theta_{j}\right)\right) \mathrm{d} \theta_{1} \cdots \mathrm{d} \theta_{n} .
$$

In order to conclude the proof of (20) we will need the following lemma:

Lemma 3. There exists a constant $C$ such that:

$$
|\hat{b}(\theta)| \leqslant C(1-\hat{a}(\theta))=C \sum_{x \in \mathbb{Z}^{d}} a(x)(1-\cos (\theta \cdot x)) .
$$

Proof (of the lemma). We have:

$$
\hat{b}(\theta)=\sum_{x \in \mathbb{Z}^{d}} b(x) \mathrm{e}^{\mathrm{i} \theta \cdot x}=\mathrm{i} \sum_{x \in \mathbb{Z}^{d}} b(x) \sin (\theta \cdot x) .
$$

Since $|b(x)| \leqslant a(x),|\hat{b}|$ is bounded by 1 .

The right-hand side of the inequality in question is a smooth function on $\mathbb{T}^{d}$. It only vanishes if $\cos (\theta \cdot x)=1$ for all $x$ such that $a(x) \neq 0$. But since the walk is assumed to be irreducible this can only happen if $\left(\theta_{1}, \ldots, \theta_{d}\right)=$ $\left(2 \sigma_{1} \pi, \ldots, 2 \sigma_{d} \pi\right)$, with $\sigma_{j} \in\{0,1\}$. We need to check that $\hat{b}$ vanishes faster at these points, and clearly, it suffices to do so at zero. Indeed, this is true because of the zero mean property of the walk which implies $\sum x b(x)=0$.

In view of the preceding lemma the expression in (27) can be estimated by:

$$
\frac{C}{(2 \pi)^{n d}}\left(\int_{\left(\mathbb{T}^{d}\right)^{n}}|\hat{g}(\theta)|^{2} H(\theta) \mathrm{d} \theta\right)^{1 / 2}\left(\int_{\left(\mathbb{T}^{d}\right)^{n}}|\hat{f}(\theta)|^{2} H(\theta) \mathrm{d} \theta\right)^{1 / 2},
$$

where for $\theta=\left(\theta_{1}, \ldots, \theta_{n}\right) \in\left(\mathbb{T}^{d}\right)^{n}, H(\theta)$ is defined by:

$$
H(\theta)=\sum_{j=1}^{n} \sum_{x \in \mathbb{Z}^{d}} a(x)\left(1-\cos \left(\theta_{j} \cdot x\right)\right) .
$$

It is easy to check that if $D_{\text {free }}^{n}$ is the Dirichlet form corresponding to $n$ free random walks on $\mathbb{Z}^{d}$ :

$$
D_{\text {free }}^{n}(u)=\frac{1}{2} \sum_{j=1}^{n} \sum_{x_{1}, \ldots, x_{n}, x_{j}^{\prime}} a\left(x_{j}^{\prime}-x_{j}\right)\left(u\left(x_{1}, \ldots, x_{j}^{\prime}, \ldots, x_{n}\right)-u\left(x_{1}, \ldots, x_{j}, \ldots, x_{n}\right)\right)^{2},
$$

then:

$$
\frac{1}{n !} D_{\text {free }}^{n}(u)=\frac{1}{(2 \pi)^{n d}} \int_{\left(\mathbb{T}^{d}\right)^{n}}|\hat{u}(\theta)|^{2} H(\theta) \mathrm{d} \theta .
$$

Hence,

$$
\left|\left\langle\mathfrak{g}, \mathfrak{L}_{0}^{b} \mathfrak{f}\right\rangle_{\mathfrak{H}}\right| \leqslant C\left[\left(\frac{1}{n !} D_{\text {free }}^{n}(f)\right)^{1 / 2}\left(\frac{1}{n !} D_{\text {free }}^{n}(g)\right)^{1 / 2}+\|\mathfrak{f}\|_{1, \text { env }}\|\mathfrak{g}\|_{1, \text { env }}\right] .
$$



(24).

We need to compare now $D_{\text {free }}^{n}(u)$ with $\mathfrak{D}^{\text {ex }}(\mathfrak{u})=\|\mathfrak{u}\|_{1, \text { env }}^{2}$ for $\mathfrak{u} \in \mathfrak{G}_{n}$ and $u\left(x_{1}, \ldots, x_{n}\right)$ defined by $\mathfrak{u}(A)$ as in

$$
\mathfrak{D}^{\operatorname{ex}}(\mathfrak{u})=\frac{1}{2 n !} \sum_{j=1}^{n} \sum_{\substack{\left(x_{1}, \ldots, x_{j}, \ldots, x_{n}\right) \in \mathcal{X}_{n} \\\left(x_{1}, \ldots, x_{j}^{\prime}, \ldots, x_{n}\right) \in \mathcal{X}_{n}}} a\left(x_{j}^{\prime}-x_{j}\right)\left(u\left(x_{1}, \ldots, x_{j}^{\prime}, \ldots, x_{n}\right)-u\left(x_{1}, \ldots, x_{j}, \ldots, x_{n}\right)\right)^{2},
$$

from which one easily checks that:

$$
\frac{1}{n !} D_{\text {free }}^{n}(u)=\mathfrak{D}^{\mathrm{ex}}(\mathfrak{u})+\sum_{|A|=n}\left(W_{1}(A)+W_{2}(A)\right) \mathfrak{u}^{2}(A),
$$

where $W_{1}(A)$ is defined after (25), and $W_{2}(A)=\sum_{x, y \in A} a(x-y)$. Using Lemma (3.7) in [16] again:

$$
\sum_{|A|=n}\left(W_{1}(A)+W_{2}(A)\right) \mathfrak{u}^{2}(A) \leqslant C n\|\mathfrak{u}\|_{1, e n v}^{2} .
$$

Thus, (20) follows by formulae (28) and (29).

We turn now to the proof of inequality (21):

$$
\begin{aligned}
\left\langle\mathfrak{g}, \mathfrak{L}_{\tau}^{1} \mathfrak{f}\right\rangle_{\mathfrak{H}}= & \sum_{|A|=n} \mathfrak{g}(A) \sum_{z \notin A} p(z)\left(\mathfrak{f}\left(\tau_{z} A\right)-\mathfrak{f}(A)\right) \\
= & -\frac{1}{2} \sum_{|A|=n} \sum_{z \notin A} a(z)\left(\mathfrak{f}\left(\tau_{z} A\right)-\mathfrak{f}(A)\right)\left(\mathfrak{g}\left(\tau_{z} A\right)-\mathfrak{g}(A)\right)+\sum_{|A|=n} \mathfrak{g}(A) \sum_{z \notin A} b(z)\left(\mathfrak{f}\left(\tau_{z} A\right)-\mathfrak{f}(A)\right) \\
= & -\frac{1}{2} \sum_{|A|=n} \sum_{z \notin A} a(z)\left(\mathfrak{f}\left(\tau_{z} A\right)-\mathfrak{f}(A)\right)\left(\mathfrak{g}\left(\tau_{z} A\right)-\mathfrak{g}(A)\right) \\
& +\sum_{|A|=n} \mathfrak{g}(A) \sum_{z \notin A} b(z) \mathfrak{f}\left(\tau_{z} A\right)+\sum_{|A|=n} \mathfrak{g}(A) \mathfrak{f}(A)\left(\sum_{z \in A} b(z)\right) .
\end{aligned}
$$

The first term in (30) can be estimated by $\sqrt{\mathfrak{D}_{\tau}^{n}(\mathfrak{f}) \mathfrak{D}_{\tau}^{n}(\mathfrak{g})}$, where using the convention $\mathfrak{f}(A)=0$, if $0 \in A$ :

$$
\mathfrak{D}_{\tau}^{n}(\mathfrak{f})=\frac{1}{2} \sum_{z} \sum_{|A|=n} a(z)(\mathfrak{f}(A-z)-\mathfrak{f}(A))^{2} .
$$

By the proof of Lemma (5.1) in [6], for every $\mathfrak{f} \in \mathfrak{G}_{n}$ we have:

$$
\mathfrak{D}_{\tau}^{n}(\mathfrak{f}) \leqslant C n\|\mathfrak{f}\|_{1, \text { env }}^{2} .
$$

Using Fourier transforms we can write:

$$
\begin{aligned}
& \sum_{|A|=n} \mathfrak{g}(A) \sum_{z \notin A} b(z) \mathfrak{f}\left(\tau_{z} A\right)=\frac{1}{(2 \pi)^{n d}} \int_{\left(\mathbb{T}^{d}\right)^{n}} \overline{\hat{g}}(\theta) \hat{f}(\theta) \hat{b}\left(\theta_{1}+\cdots+\theta_{n}\right) \mathrm{d} \theta, \\
& \mathfrak{D}_{\tau}^{n}(\mathfrak{f})=\frac{1}{(2 \pi)^{n d}} \int_{\left(\mathbb{T}^{d}\right)^{n}}|\hat{f}(\theta)|^{2}\left(1-\hat{a}\left(\theta_{1}+\cdots+\theta_{n}\right)\right) \mathrm{d} \theta .
\end{aligned}
$$

Since by Lemma 3 we have $|\hat{b}(\theta)| \leqslant C(1-\hat{a}(\theta))$, it follows that:

$$
\left|\sum_{|A|=n} \mathfrak{g}(A) \sum_{z \notin A} b(z) \mathfrak{f}\left(\tau_{z} A\right)\right| \leqslant C \mathfrak{D}_{\tau}^{1 / 2}(\mathfrak{f}) \mathfrak{D}_{\tau}^{1 / 2}(\mathfrak{g}) .
$$


Hence, the estimate (21) follows by formulae (26) and (30)-(32).

On the other hand:

$$
\begin{aligned}
\left|\left\langle\mathfrak{g}, \mathfrak{L}_{\tau}^{2} \mathfrak{f}\right\rangle_{\mathfrak{H}}\right| & =\left|\sum_{|A|=n} \mathfrak{g}(A) \sum_{z \in A} p(z)\left(\mathfrak{f}\left(\tau_{z} A\right)-\mathfrak{f}(A)\right)\right| \\
& \leqslant\left(\sum_{|A|=n} \mathfrak{g}^{2}(A)\left(\sum_{z \in A} p(z)\right)\right)^{1 / 2}\left(\sum_{|A|=n} \sum_{z \in A} p(z)\left(\mathfrak{f}\left(\tau_{z} A\right)-\mathfrak{f}(A)\right)^{2}\right)^{1 / 2} \\
& \leqslant\left(2 \sum_{|A|=n} \mathfrak{g}^{2}(A) W_{1}(A)\right)^{1 / 2}\left(\sum_{|A|=n} \sum_{z \in A} p(z)\left(\mathfrak{f}\left(\tau_{z} A\right)-\mathfrak{f}(A)\right)^{2}\right)^{1 / 2}
\end{aligned}
$$

Therefore using (31) and Lemma (3.7) in [16] once more:

$$
\left|\left\langle\mathfrak{g}, \mathfrak{L}_{\tau}^{2} \mathfrak{f}\right\rangle_{\mathfrak{H}}\right| \leqslant C \sqrt{n}\|\mathfrak{f}\|_{1, \text { env }}\|\mathfrak{g}\|_{1, \text { env }},
$$

which concludes the proof of the theorem.

We can proceed now with the proof of Lemma 1. The original proof in [6] relies on the following facts:

- If $u_{\lambda}$ is the solution to the resolvent equation: $\lambda u_{\lambda}-\mathcal{L} u_{\lambda}=-\psi$ then:

$$
\lim _{\lambda \downarrow 0}\left\|\mathcal{L} u_{\lambda}-\psi\right\|_{-1, \rho}=0 .
$$

This follows by the sector condition of the generator for the mean zero asymmetric simple exclusion process and it is proved in [18].

- The convergence takes place in $H_{-1,0, \rho}$ as well, so that for any $\varepsilon>0$ we can find a $\lambda_{0}$ such that:

$$
\left\|\mathcal{L} u_{\lambda_{0}}-\psi\right\|_{-1,0, \rho} \leqslant \varepsilon / 2
$$

As

$$
\left\|\mathcal{L} u_{\lambda}-\psi\right\|_{-1,0, \rho}^{2}=\sum_{n \leqslant n_{0}}\left\|\pi_{n}\left(\mathcal{L} u_{\lambda}-\psi\right)\right\|_{-1,0, \rho}^{2}+\sum_{n>n_{0}}\left\|\pi_{n} \mathcal{L} u_{\lambda}\right\|_{-1,0, \rho}^{2},
$$

this consists of showing the following facts:

(i) For any $u \in L^{2}\left(\mu_{\rho}\right) \cap H_{-1,0, \rho}$ we have:

$$
\left\|\pi_{n} u\right\|_{-1,0, \rho} \leqslant C(n)\|u\|_{-1, \rho}
$$

hence, finite sums converge to zero. This follows by (31) with $C(n)=C \sqrt{n}$.

(ii) The tail of the sum can be controlled, i.e.

$$
\left\|\pi_{n} \mathcal{L} u\right\|_{-1,0, \rho} \leqslant C_{n} \sum_{j=-1}^{+1}\left\|\pi_{n+j} u\right\|_{1,0, \rho},
$$

and $\left\|\pi_{n} u_{\lambda}\right\|_{1,0, \rho}$ decay rapidly with $n$. The estimate (34) that was established by reversibility in [6] now follows from Theorem 4 with $C_{n}=C n$. On the other hand, if we define:

$$
\|u\|_{ \pm 1, k}^{2}=\sum_{n \geqslant 0} n^{2 k}\left\|\pi_{n} u\right\|_{ \pm 1,0, \rho}^{2},
$$

it is shown in [8] that from the estimates proved in Theorem 4 it follows that:

$$
\left\|u_{\lambda}\right\|_{1, k} \leqslant C(k)\|\mid \psi\|_{-1, k}
$$

for any positive integer $k$. Hence, since $\psi$ is local the components of high degrees of $u_{\lambda}$ exhibit a faster than polynomial decay. 
- We can find a local $g$ such that:

$$
\left\|\mathcal{L}\left(u_{\lambda_{0}}-g\right)\right\|_{-1,0, \rho} \leqslant \varepsilon / 2 .
$$

This follows again from the fast decay of $\left\|\pi_{n} \mathcal{L} u_{\lambda}\right\|_{-1,0, \rho}$, which reduces the problem to approximating on finite degrees (cf. [6] for a detailed proof).

\section{Proof of Theorem 3}

Using duality we can express $\Delta_{\ell}(\rho)$ as:

$$
\Delta_{\ell}(\rho)=\rho(1-\rho) \lim _{\lambda \downarrow 0}\left\langle(\lambda-\mathfrak{L}(\rho))^{-1} \mathfrak{w}_{\ell},(1+R) \mathfrak{w}_{\ell}\right\rangle_{\mathfrak{H}},
$$

where

$$
\mathfrak{w}_{\ell}=\sum_{x} p(x)(x \cdot \ell) e_{\{x\}},
$$

and $R \mathfrak{f}(A)=\mathfrak{f}(-A)$.

If we define $\Phi_{\ell}=\frac{1+R}{2} \mathfrak{w}$, it is immediate that $\phi_{\ell} \mapsto \sqrt{\chi(\rho)} \Phi_{\ell}$, and thus:

$$
\left\|\phi_{\ell}\right\|_{-1,0, \rho}^{2}=\rho(1-\rho)\left\|\Phi_{\ell}\right\|_{-1, e n v}^{2} .
$$

The proof of Theorem 3 consists of the following two lemmata:

\section{Lemma 4.}

$$
\lim _{\lambda \downarrow 0}\left\langle(\lambda-\mathfrak{L}(0))^{-1} \mathfrak{w}_{\ell},(1+R) \mathfrak{w}_{\ell}\right\rangle_{\mathfrak{H}}=\left\|\Phi_{\ell}\right\|_{-1, e n v}^{2} .
$$

\section{Lemma 5.}

$$
\limsup _{\rho \downarrow 0}\left\langle\left[(\lambda-\mathfrak{L}(\rho))^{-1}-(\lambda-\mathfrak{L}(0))^{-1}\right] \mathfrak{w}_{\ell},(1+R) \mathfrak{w}_{\ell}\right\rangle_{\mathfrak{H}}=0 .
$$

Proof (of Lemma 4). Notice that when $\rho=0$ the part of the generator that changes the degree vanishes, hence the equation:

$$
\lambda \mathfrak{u}_{\lambda}-\mathfrak{L}(0) \mathfrak{u}_{\lambda}=\mathfrak{w}_{\ell}
$$

can be solved in $\overline{\mathfrak{G}_{1}}$. Note that $\mathfrak{L}(0)=\mathfrak{L}_{0}+\mathfrak{L}_{0}^{b}+\mathfrak{L}_{\tau}^{1}$, and that if we use the convention $\mathfrak{f}(x)=\mathfrak{f}(\{x\})$ on $\mathfrak{G}_{1}$, we have:

$$
\mathfrak{L}_{0}^{b} \mathfrak{f}(x)=\sum_{y \neq 0, x} b(y-x)(\mathfrak{f}(y)-\mathfrak{f}(x)),
$$

while

$$
\mathfrak{L}_{\tau}^{1} \mathfrak{f}(x)=\sum_{y \neq 0, x} p(y)(\mathfrak{f}(x-y)-\mathfrak{f}(x))=\sum_{y \neq x, 0} p(x-y)(\mathfrak{f}(y)-\mathfrak{f}(x)) .
$$

Hence, we can combine $\mathfrak{L}_{0}^{b}$ and $\mathfrak{L}_{\tau}^{1}$ to get:

$$
\mathfrak{L}_{0}^{b}+\mathfrak{L}_{\tau}^{1}=\mathfrak{L}_{0} \Rightarrow \mathfrak{L}(0)=2 \mathfrak{L}_{0}, \quad \text { on } \mathfrak{G}_{1} .
$$

In particular $\mathfrak{L}(0)$ commutes with $R$ on $\mathfrak{G}_{1}$ and thus: 


$$
\begin{aligned}
\left\langle(\lambda-\mathfrak{L}(0))^{-1} \mathfrak{w}_{\ell},(1+R) \mathfrak{w}_{\ell}\right\rangle_{\mathfrak{H}} & =2\left\langle(\lambda-\mathfrak{L}(0))^{-1} \Phi_{\ell}, \Phi_{\ell}\right\rangle_{\mathfrak{H}}=\left\langle\left(\frac{\lambda}{2}-\mathfrak{L}_{0}\right)^{-1} \Phi_{\ell}, \Phi_{\ell}\right\rangle_{\mathfrak{H}} \\
& \rightarrow\left\|\Phi_{\ell}\right\|_{-1, \text { env }}^{2}, \quad \text { as } \lambda \rightarrow 0 .
\end{aligned}
$$

Proof (of Lemma 5). Since $R$ is a unitary isomorphism in $\mathfrak{H}$ that commutes with $\mathfrak{L}_{0}$ it preserves the norms $\|\cdot\|_{1, \text { env }}$ and $\|\cdot\|_{-1, e n v}$. Therefore,

$$
\left\|\frac{1+R}{2} \mathfrak{w}_{\ell}\right\|_{-1, \text { env }}^{2}+\left\|\frac{1-R}{2} \mathfrak{w}_{\ell}\right\|_{-1, \text { env }}^{2}=\left\|\mathfrak{w}_{\ell}\right\|_{-1, \text { env }}^{2},
$$

which by [8] (cf. discussion before Lemma 4.4) is finite.

Let us now consider the resolvent equation:

$$
\lambda U_{\lambda, \rho}-\mathfrak{L}(\rho) U_{\lambda, \rho}=V .
$$

If we multiply both sides by $U_{\lambda, \rho}$ and integrate we get: $\left\|(\lambda-\mathfrak{L}(\rho))^{-1} V\right\|_{1, \rho} \leqslant\|V\|_{-1, \rho}$. On the other hand if we subtract (37) for $\rho=0$ from (37) for the general $\rho>0$ we have:

$$
(\lambda-\mathfrak{L}(\rho))\left(U_{\lambda, \rho}-U_{\lambda, 0}\right)=(\mathfrak{L}(0)-\mathfrak{L}(\rho)) U_{\lambda, 0} .
$$

It is easy to check that:

$$
\mathfrak{L}(0)-\mathfrak{L}(\rho)=\rho\left(2 \mathfrak{L}_{0}^{b}+\mathfrak{L}_{\tau}^{1}-\mathfrak{L}_{\tau}^{2}\right)+\sqrt{\chi(\rho)}\left(\mathfrak{L}^{+}+\mathfrak{L}^{-}\right) .
$$

Let us take now $V=\mathfrak{w}_{\ell}$. As we mentioned in the proof of Lemma $4 U_{\lambda, 0} \in \overline{\mathfrak{G}_{1}}$. Therefore, it follows immediately by Theorem 4 that:

$$
\left\|(\mathfrak{L}(0)-\mathfrak{L}(\rho)) U_{\lambda, 0}\right\|_{-1, \rho} \leqslant C \sqrt{\rho}\left\|U_{\lambda, 0}\right\|_{1, \text { env }} \leqslant C \sqrt{\rho}\left\|\mathfrak{w}_{\ell}\right\|_{-1, \text { env }} .
$$

Hence, using (38) and the preceding estimate we have:

$$
\begin{aligned}
\left|\left\langle U_{\lambda, \rho}-U_{\lambda, 0},(1+R) \mathfrak{w}_{\ell}\right\rangle_{\mathfrak{H}}\right| & \leqslant\left\|U_{\lambda, \rho}-U_{\lambda, 0}\right\|_{1, \rho}\left\|(1+R) \mathfrak{w}_{\ell}\right\|_{-1, \rho} \\
& \leqslant 2\left\|(\mathfrak{L}(0)-\mathfrak{L}(\rho)) U_{\lambda, 0}\right\|_{-1, \rho}\left\|\mathfrak{w}_{\ell}\right\|_{-1, e n v} \\
& \leqslant C \sqrt{\rho}\left\|\mathfrak{w}_{\ell}\right\|_{-1, \text { env }}^{2},
\end{aligned}
$$

which tends to zero uniformly in $\lambda$, as $\rho \rightarrow 0$.

\section{Remarks}

It is interesting to compare the self-diffusion coefficient $D(\rho)$ of a tagged particle in mean zero asymmetric simple exclusion process to $D^{s}(\rho)$, the one in its symmetrized version, i.e. the simple exclusion with generator $\mathcal{S}=1 / 2\left(\mathcal{L}+\mathcal{L}^{*}\right)$. As pointed out in [16] using a variational argument the former is always greater than or equal the latter. We obtain the following corollary to Theorem 3:

Corollary 1. For any $\ell \in \mathbb{R}^{d}$ :

$$
\ell \cdot\left(D(\rho)-D^{S}(\rho)\right) \ell=2 \lim _{\lambda \downarrow 0}\left\|(\lambda-\mathcal{L})^{-1} \omega_{\ell}-(\lambda-\mathcal{S})^{-1} \psi_{\ell}\right\|_{1, \rho}^{2} .
$$

Hence, unless $\ell$ is orthogonal to the support of $b(\cdot)$ we have $\ell \cdot\left(D(\rho)-D^{s}(\rho)\right) \ell>0$ for sufficiently small values of $\rho$. 
By (5), for every $\ell \in \mathbb{R}^{d}$ we have:

$$
\ell \cdot\left(D(\rho)-D^{s}(\rho)\right) \ell=2 \lim _{\lambda \downarrow 0}\left((\lambda-\mathcal{L})^{-1} \omega_{\ell}, R \omega_{\ell}\right\rangle_{\rho}-\left\langle(\lambda-\mathcal{S})^{-1} \psi_{\ell}, R \psi_{\ell}\right\rangle_{\rho} .
$$

If we set $u_{\lambda}=(\lambda-\mathcal{L})^{-1} \omega_{\ell}$ and $v_{\lambda}=(\lambda-\mathcal{S})^{-1} \psi_{\ell}$ then:

$$
\begin{aligned}
\left\|u_{\lambda}-v_{\lambda}\right\|_{1, \rho}^{2} & =\left\|u_{\lambda}\right\|_{1, \rho}^{2}+\left\|v_{\lambda}\right\|_{1, \rho}^{2}-2\left\langle u_{\lambda},(-\mathcal{S}) v_{\lambda}\right\rangle_{\rho} \\
& =\left\langle\omega_{\ell}, u_{\lambda}\right\rangle_{\rho}+\left\langle\psi_{\ell}, v_{\lambda}\right\rangle_{\rho}-2\left\langle\psi_{\ell}, u_{\lambda}\right\rangle_{\rho}-\lambda\left\|u_{\lambda}-v_{\lambda}\right\|_{L^{2}\left(\mu_{\rho}\right)}^{2} \\
& =\left\langle u_{\lambda}, R \omega_{\ell}\right\rangle_{\rho}-\left\langle v_{\lambda}, R \psi_{\ell}\right\rangle_{\rho}-\lambda\left\|u_{\lambda}-v_{\lambda}\right\|_{L^{2}\left(\mu_{\rho}\right)}^{2} \\
& \rightarrow \frac{1}{2} \ell \cdot\left(D(\rho)-D^{s}(\rho)\right) \ell, \quad \text { as } \lambda \downarrow 0 .
\end{aligned}
$$

Notice now that since $R$ commutes with $\mathcal{S}, v_{\ell}$ inherits the antisymmetry under $R$ from $\psi_{\ell}$. On the other hand $\phi_{\ell}$ is symmetric under $R$ and thus orthogonal to $v_{\ell}$. Hence, we obtain from (9) that:

$$
\Delta_{\ell}(\rho)=\lim _{\lambda \downarrow 0}\left\langle u_{\lambda}, \phi_{\ell}\right\rangle_{\rho}=\lim _{\lambda \downarrow 0}\left\langle u_{\lambda}-v_{\lambda}, \phi_{\ell}\right\rangle_{\rho},
$$

and the corollary follows from Theorem 3.

It is also interesting to recall some regularity properties of the self-diffusivity as a function of $\rho$. Since $D(\rho)$ appears as a coefficient in parabolic equations governing the non-equilibrium evolution of the density in systems where the number of particles is preserved under the dynamics (cf. for instance [14]), it is important to establish first its regularity in $\rho$ in order to conclude the existence and regularity of the solutions to these equations. Landim, Olla and Varadhan have proved (cf. [8]) that the estimates (19)-(22) guarantee $D(\rho)$ to be of class $C^{\infty}$ on $[0,1]$. We remind the reader that the proof of smoothness can be carried out by reparametrising as $\rho=\sin ^{2} t$, then using the estimates (19)-(22) to prove the smoothness of $D$ as a function of $t$. This immediately implies smoothness for $\rho \in(0,1)$, while the observation that the odd derivatives of $D$ vanish at the boundary takes care of the smoothness there. The authors prove these estimates in the symmetric case (cf. [7]) and they also prove that $D(\rho)$ is of class $C^{\infty}$ on $(0,1]$ in the asymmetric case when $d \geqslant 3$ (cf. [8]). Hence the following immediate corollary to their results and Theorem 19, extends, in the mean zero asymmetric case, the smoothness of $D$ to zero as well:

Corollary 2. If $d \geqslant 3$ the self-diffusion coefficient $D(\rho)$ of a tagged particle in a mean zero asymmetric simple exclusion process is of class $C^{\infty}$ in $\rho$ on $[0,1]$.

The technique we employ here to compute the mobility is fairly general. It is essentially in the spirit of the entropy method introduced in [2], only the process in question here has different dynamics from the reference stationary process, rather than different initial state. The only ad hoc for the simple exclusion proof is that of Eq. (17):

$$
\lim _{\gamma \rightarrow 0} \gamma^{-1} \lambda_{\rho}^{*}(\gamma f)=\left\langle f, \mu_{\rho}\right\rangle
$$

and in principle, the same arguments can go through for any system satisfying this eigenvalue estimate. Models for which the generator of the dynamics satisfies a spectral gap property provide examples for which (17) is always true (cf. Appendix 3.1 in [3]); It is worth noting at this point that in the context of this further assumption (spectral gap) Komorowski and Olla prove in a recent article ([5]) the existence of an asymptotic velocity for the tagged particle in the perturbed dynamics and check the validity of the Einstein relation for the symmetric simple exclusion process with absorption-desorption. By generalising their argument, they conclude the validity of the Einstein relation in a quite general set-up of models with reversible dynamics satisfying the spectral gap property, and they also provide an example of a non-reversible system for which the Einstein relation is satisfied. 


\section{Acknowledgements}

The greatest part of this work was carried out when the author was visiting the Forschungsinstitut für Mathematik at ETH, Zürich. The author wishes to express his gratitude to the people at FIM for their warm Swiss hospitality, and Professor Alain-Sol Sznitman in particular for his useful comments and suggestions. This research has also been supported by a Marie Curie Fellowship of the European Community Programme "Improving Human Potential" under the contract number HPMF-CT-2002-01610.

\section{References}

[1] P.A. Ferrari, S. Goldstein, J.L. Lebowitz, Diffusion, mobility and the Einstein relation, in: J. Fritz, A. Jaffe, D. Száz (Eds.), Statistical Physics and Dynamical Systems, Birkhäuser, Boston, 1985, pp. 405-441.

[2] M.Z. Guo, G.C. Papanicolaou, S.R.S. Varadhan, Nonlinear diffusion limit for a system with nearest neighbor interactions, Comm. Math. Phys. 118 (1988) 31-59.

[3] C. Kipnis, C. Landim, Scaling Limits of Interacting Particle Systems, Grundlehren Math. Wiss., Springer-Verlag, 1999.

[4] C. Kipnis, S.R.S. Varadhan, Central limit theorem for additive functionals of reversible Markov processes and applications to simple exclusion, Comm. Math. Phys. 106 (1986) 1-19.

[5] T. Komorowski, S. Olla, On mobility and Einstein relation for tracers in time-mixing random environments, J. Statist. Phys., in press, preprint available at http://www.ceremade.dauphine.fr/ olla.

[6] C. Landim, S. Olla, S.R.S. Varadhan, Finite dimensional approximation of the self-diffusion coefficient for the exclusion process, Ann. Probab. 30 (2) (2002) 483-508.

[7] C. Landim, S. Olla, S.R.S. Varadhan, Symmetric simple exclusion process: regularity of the self diffusion coefficient, Comm. Math. Phys. 224 (1) (2001) 307-321.

[8] C. Landim, S. Olla, S.R.S. Varadhan, Asymptotic behavior of a tagged particle in simple exclusion processes, Bol. Soc. Brasil. Mat. (N.S.) 31 (3) (2000) 241-275.

[9] C. Landim, S. Olla, S.B. Volchan, Driven tracer particle in 1 dimensional symmetric simple exclusion, Comm. Math. Phys. 192 (2) (1998) 287-307.

[10] C. Landim, H.T. Yau, Fluctuation-dissipation equation of asymmetric simple exclusion processes, Probab. Theory Related Fields 108 (1997) 321-356.

[11] J.L. Lebowitz, H. Rost, The Einstein relation for the displacement of a test particle in a random environment, Stochastic Process. Appl. 54 (1994) 183-196.

[12] T. Liggett, Interacting Particle Systems, Grundlehren Math. Wiss., Springer-Verlag, New York, 1985.

[13] M. Loulakis, Einstein relation for a tagged particle in simple exclusion processes, Comm. Math. Phys. 229 (2) (2002) $347-367$.

[14] J. Quastel, Diffusion of color in the simple exclusion process, Comm. Pure Appl. Math. 45 (6) (1992) 623-679.

[15] E. Saada, A limit theorem for the position of a tagged particle in a simple exclusion process, Ann. Probab. 15 (1987) 375-381.

[16] S. Sethuraman, H.T. Yau, S.R.S. Varadhan, Diffusive limit of a tagged particle in asymmetric simple exclusion processes, Comm. Pure Appl. Math. 53 (2000) 972-1006.

[17] R.M. Sued, Regularity properties of the diffusion coefficient for a mean zero exclusion process, IMPA Thesis, available at: http://www.preprint.impa.br.

[18] S.R.S. Varadhan, Self diffusion of a tagged particle in equilibrium for asymmetric mean zero random walk with simple exclusion, Ann. Inst. H. Poincaré Probab. Statist. 31 (1995) 273-285. 